\title{
Optimal Bidding in Sequential Auctions
}

\author{
SHMUEL S. OREN \\ Xerox Corporation, Palo Alto, California, and Stanford University, \\ Stanford, California \\ MICHAEL H. ROTHKOPF \\ Xerox Corporation, Palo Alto, California
}

(Received original April 11, 1974; final, March 3, 1975)

\begin{abstract}
A bidder's strategy in one auction may affect his competitors' behavior in subsequent auctions. When this occurs, bidding in a sequence of auctions can be modeled fruitfully as a multistage control process. This paper presents such a model. In it the control is the bidder's strategy, the state characterizes the competitors' behavior and the state transition represents the competitors' reaction to the bidder's strategy. Dynamic programming is used to derive the infinite horizon optimal bidding strategy. We show that in steady state this optimal strategy generalizes a previous result for equilibrium bidding strategy in 'one-shot' auctions.
\end{abstract}

$\mathbf{M}$

$\mathrm{UCH}$ of the theory of competitive bidding and all of the early developments in that theory dealt with 'one-shot' situations, that is, bidding situations in which it is appropriate for the bidder to attempt to maximize his expected profit from the present auction or simultaneous group of auctions. Recently, a number of models for optimum bidding in sequential auctions have been developed. ${ }^{[1-3,6-8,13-15]}$ All of these deal with the internal effects within the bidding firm of winning or losing auctions. With the exception of a little-known and specialized paper of Banerjee and Ghosh, ${ }^{[2]}$ they all share the assumption that the competition will not react in later auctions to what the bidder has done in earlier auctions (see Note 1). At times this must be a tenuous assumption in a field that is filled with literature suggesting how bidders should use information about the past behavior of competitors to determine their bids in present auctions. Therefore, we have built a model of bidding in sequential auctions in which we assume a bidder's competitors will react to his previous bids.

One possible approach is to model the bidding process as a dynamic multiplayer noncooperative game. This, however, seems difficult. Our approach is less ambitious. We assume that bidders develop a behavioral model of how their competitiors will react. This assumption leads us to 
model the sequential bidding problem as a multistage control process, in which the control is the bidder's strategy and the state characterizes the collective behavior of the competitors. In this model the state transition represents the competitors' reaction to the bidder's policy. We use dynamic programming to derive an equation for the optimal infinite horizon bidding strategy. When this equation is solved for a generalization of a previous model of 'one-shot' auctions, ${ }^{[9]}$ the formula for the optimal oneshot policy is modified by the inclusion of a term that depends upon the magnitude of competitive reaction, the time between auctions, and the discount rate.

\section{A DYNAMIC MODEL OF BIDDING IN SEQUENTIAL AUCTIONS}

Consider a bidder who faces an infinite sequence of sealed-bid auctions. In each auction his expected profit from that auction depends upon the bidding policy of the rest of the industry and upon his own bidding policy. He would like to choose his own policy to maximize his expected profit from the immediate auction. However, he must keep in mind that his actions in the present auction will be observed by his competitors and will influence their behavior in future auctions. Therefore, it is in his interest to choose a bidding policy that will maximize his expected present value of profits in the present auction and all future auctions. It is this process that we propose to model.

Let $\boldsymbol{P}(k)$ be an $n$-dimensional vector where $n$ is the total number of bidders and $P_{i}(k)$, the $i$ th component of $\boldsymbol{P}(k)$, is a scalar representing the bidding policy of the $i$ th bidder in the $k$ th auction. We assume the expected reward of the $i$ th bidder in the $k$ th auction, denoted by $E_{i}(k)$, to be a fixed function of $\boldsymbol{P}(k)$. Furthermore, this reward function is assumed to have the form

$$
E_{i}(k)=E_{i}\left[Q_{i}(k), P_{i}(k)\right],
$$

where $Q_{i}(k)$ is a scalar function of all the components of $\boldsymbol{P}(k)$ excluding $P_{i}(k)$ such that if $P_{j}(k)=P$ for all $j \neq i$, then $Q_{i}(k)=P$. The reward function $E_{i}$ summarizes all of the internal effects on the bidder of winning or losing a particular auction, along with his likelihood of winning.

Under these assumptions the $i$ th bidder may view $Q_{i}(k)$ as a variable representing the aggregate bidding policy of the rest of the trade. Furthermore, the effect of his present bidding policy on the future behavior of the rest of the trade may be encoded in terms of the change in $Q_{i}(k)$. In particular, we shall assume that at any auction $k+1$

$$
Q_{i}(k+1)=f_{i}\left[Q_{i}(k), P_{i}(k)\right] .
$$

This assumption implies that the aggregate policy of the trade, as viewed by the $i$ th bidder in the $(k+1)$ th auction depends on this aggregate policy 
and the policy of the $i$ th bidder in the $k$ th auction. Equation (2) may be viewed as a behavioral assumption on how the $i$ th bidder views the dynamics of the trade. However, it may also be regarded as an approximation to the update of $Q_{i}(k)$ that could result from a game-theoretic approach.

The above assumptions enable the $i$ th bidder to view the process of bidding in sequential auction as a multistage control process (see Bryson and $\mathrm{Ho}^{[4]}$ ), where $P_{i}(k)$ is the control, $Q_{i}(k)$ is a state variable, and equation (2) is the state transition function. Bidder $i$ 's objective is to determine a control sequence that will maximize his present value of the rewards over an infinite horizon. Let $D_{i}$ be the discount factor of the $i$ th bidder; i.e., $D_{i}=\exp \left(-r_{i} t\right)$, where $t$ is the time between auctions and $r_{i}$ is the continuous discount rate of the $i$ th bidder. Then bidder $i$ 's problem is to maximize $\sum_{k=0}^{k=\infty} D_{i}{ }^{k} E_{i}(k)$, where $E_{i}(k)$ is given by $(1)$.

We can solve this problem using dynamic programming. Let

$$
V_{i}\left(Q_{i}, j\right)=\max _{\left\{P_{i}(k)\right\} j_{j}^{\infty}} \sum_{k=j}^{k=\infty} D_{i}^{(k-j)} E_{i}\left[Q_{i}(k), P_{i}(k)\right],
$$

subject to

$$
Q_{i}(k+1)=f_{i}\left[Q_{i}(k), P_{i}(k)\right], \quad Q_{i}(j)=Q_{i} .
$$

One can easily show that for $D_{i}<1$,

$$
V_{i}\left(Q_{i}, j\right)=V_{i}\left(Q_{i}, j+1\right)=V_{i}\left(Q_{i}\right) .
$$

Thus the argument $j$ can be suppressed, and by the 'principle of optimality' we have

$$
V_{i}\left(Q_{i}\right)=\max _{P}\left\{E_{i}\left(Q_{i}, P\right)+D_{i} V_{i}\left[f_{i}\left(Q_{i}, P\right)\right]\right\} .
$$

For notational convenience we shall temporarily omit the subscripts $i$. Let $\hat{P}(Q)$ be the value of $P$ that maximizes the right-hand side of $(5)$ for a given value of $Q$. Then

$$
V(Q)=E[Q, \hat{P}(Q)]+D V[f(Q, \hat{P}(Q))] .
$$

If we assume $E(Q, P)$ and $f(Q, P)$ are differentiable with respect to $P$, $\hat{P}(Q)$ is a stationary point satisfying the necessary condition

$$
\left.\left\{[\partial E(Q, P) / \partial P]+\left.D[d V(\xi) / d \xi]\right|_{\xi=f(Q, P)} \partial f(Q, P) / \partial P\right\}\right|_{P=\hat{P}(Q)}=0
$$

for any given $Q$.

Substituting $\xi$ for $Q$ in (6) and differentiating with respect to $\xi$ yield

$$
\begin{aligned}
& d V(\xi) / d \xi=\{[\partial E(\xi, \eta) / \partial \xi] \\
& \left.+\left.D[d V(\mu) / d \mu]\right|_{\mu=f(\xi, \eta)} \partial f(\xi, \eta) / \partial \xi\right\}\left.\right|_{\eta=\hat{P}(\xi)}+\{[\partial E(\xi, \eta) / \partial \eta] \\
& \left.+\left.D[d V(\mu) / d \mu]\right|_{\mu=f(\xi, \eta)} \partial f(\xi, \eta) / \partial \eta\right\}\left.\right|_{\eta=\hat{P}(\xi)} d \hat{P}(\xi) / d \xi .
\end{aligned}
$$

The second part of (8) is zero by (7). Solving (7) for $D[d V(\xi) / d \xi]$ at $\xi=f[Q, P(Q)]$ and substituting it into the remainder of (8) give

$$
\begin{aligned}
d V(\xi) / d \xi=\{[\partial E(\xi, \eta) / \partial \xi] & \\
- & {[\partial E(\xi, \eta) / \partial \eta][\partial f(\xi, \eta) / \partial \xi] /[\partial f(\xi, \eta) / \partial \eta]\}\left.\right|_{\eta=\hat{P}(\xi) .} }
\end{aligned}
$$


Substituting back into (7) and restoring the subscript $i$ yield

$$
\begin{aligned}
& {\left[\left[\partial E_{i}\left(Q_{i}, \eta\right) / \partial \eta\right]+\right.} D_{i}\left[\partial f_{i}\left(Q_{i}, \eta\right) / \partial \eta\right] \cdot \\
& \cdot\left\{\left[\partial E_{i}(\xi, \eta) / \partial \xi\right]-\left[\partial E_{i}(\xi, \eta) / \partial \eta\right]\right. \\
&\left.\left.\cdot\left[\partial f_{i}(\xi, \eta) / \partial \xi / \partial f_{i}(\xi, \eta) / \partial \eta\right]\right\}\right]\left.\right|_{\eta=\hat{P} i(Q i), \xi=f i[Q i, \hat{P} i(Q i)]}=0 .
\end{aligned}
$$

Equation (10) is a necessary condition for the optimal strategy $\hat{P}_{i}\left(Q_{i}\right)$. According to this strategy, bidder $i$ 's optimal policy in any auction will be

$$
P_{i}^{*}(k)=\hat{P}_{i}\left[Q_{i}(k)\right] \text {. }
$$

Note that $\hat{P}_{i}\left[Q_{i}(k)\right]$ is bidder $i$ 's optimal policy given his assumption that the trade's behavior is represented by $Q_{i}(k)$ and $(2)$. This result is true independently of whether bidder $i$ 's previous policies were optimal and of whether the trade has followed the assumed reaction function in the past.

\section{OPTIMAL EQUILIBRIUM POLICY FOR IDENTICAL BIDDERS}

To obtain more specific results, we now consider the special case of identical bidders, i.e., when $f_{i}, E_{i}$, and $D_{i}$ are the same for all $i$. Conceivably there exists in this case an optimal equilibrium policy $P^{*}$ such that, if all the bidders use this policy, it is optimal for each of them to keep using it. If so, then $\hat{P}_{i}\left(P^{*}\right)=P^{*}$ for all $i$. Furthermore, for the reaction function $f_{i}$ to be consistent dynamically with such behavior, it has to satisfy

$$
f_{i}\left(P^{*}, P^{*}\right)=P^{*} \text {. }
$$

Substituting these two conditions in (10) and eliminating the subscript $i$ result in the following equation for $P^{*}$ :

$$
\begin{aligned}
\{[\partial E(\xi, \eta) / \partial \eta]+D[ & (\partial E(\xi, \eta) / \partial \xi)(\partial f(\xi, \eta) / \partial \eta) \\
& -(\partial E(\xi, \eta) / \partial \eta)[\partial f(\xi, \eta) / \partial \xi]]\}\left.\right|_{\xi=\eta=P \bullet}=0 .
\end{aligned}
$$

For the policy $P^{*}$ to be a meaningful equilibrium policy, it should be a stable equilibrium in the sense that small deviations from this policy will create incentives that will drive the trade back to equilibrium. This stability condition imposes an additional restriction on the reaction function. In particular, $f$ has to be such that for sufficiently small $\Delta P$

$$
\left|f\left[P^{*}+\Delta P, \hat{P}\left(P^{*}+\Delta P\right)\right]-P^{*}\right| \leqq \theta|\Delta P| \text { for some } \theta<1 .
$$

For $\Delta P \rightarrow 0$, equation (14) becomes

$$
|[[\partial f(\xi, \eta) / \partial \xi]+[\partial f(\xi, \eta) / \partial \eta][d \hat{P}(\xi) / d \xi]]|_{\xi=\eta=P^{*}} \mid<1 .
$$

In view of the above discussion, it is clearly desirable from a game-theoretic point of view to choose a reaction function $f$ satisfying (12), (13), and (15). Using such a response function leads to an equilibrium policy that is consistent with the game-theoretic approach, in which all bidders are assumed 
to optimize their policy while considering that their competitors do the same.

\section{REACTION FUNCTIONS}

In this section we discuss two specific forms for the reaction function $f$ in our model and their implications.

The functional form we have chosen to consider initially is

$$
f\left(Q_{i}, P_{i}\right)=Q_{i}-\alpha\left[(1 / n)-p_{i}\right]\left(P_{i}-Q_{i}\right) .
$$

In (16) $Q_{i}$ and $P_{i}$ are the respective policies of the trade and bidder $i, n$ is the total number of bidders, $\alpha$ is a scalar parameter, and $p_{i}$ is the probability of bidder $i$ winning. The probability $p_{i}$ is, in general, a function of the number of bidders $n$ and the policies $Q_{i}$ and $P_{i}$.

The reaction function defined by (16) corresponds to an assumption that the trade reaction to a deviation from trade policy by bidder $i$ will be proportional to the product of the extent of the deviation and the extent by which bidder $i$ deviates from his 'fair' share, $1 / n$, of the market. The constant $\alpha$ is, of course, the constant of proportionality. We assume it to be positive. With this definition, in the case of identical bidders, an equilibrium policy implies $Q_{i}=P_{i}=P^{*}$ and $p_{i}=1 / n$. Thus (16) satisfies condition (12).

Figure 1 illustrates $f\left(Q_{i}, P_{i}\right)$ as a function of $P_{i}$ for a given $Q_{i}$. If we assume that $P_{i}=0$ implies $p_{i}=1$, the function begins at $P_{i}=0$ with the value $f\left(Q_{i}, 0\right)=Q_{i}[1-\alpha(n-1) / n]$. Then it rises to a maximum of $Q_{i}$ at $P_{i}=Q_{i}$ and declines.

This choice of reaction function has several important consequences. One can easily verify that for this function,

and

$$
\partial f(\xi, \eta) /\left.\partial \eta\right|_{\xi=\eta=P^{*}}=0
$$

$$
\partial f(\xi, \eta) /\left.\partial \xi\right|_{\xi=\eta=P^{*}}=1 \text {. }
$$

Thus, if we substitute (17) and (18) in (13) and assume $D<1$, we obtain the equilibrium condition

$$
\partial E\left(P^{*}, \eta\right) /\left.\partial \eta\right|_{\eta=P^{*}}=0 \text {. }
$$

This is the same condition as for the 'one-shot' situation. Substituting (17) and (18) in the left-hand side of (15) indicates, however, that $P^{*}$ satisfying (19) is an unstable equilibrium. This instability has disconcerting implications with respect to the dynamics of the trade policy. As illustrated by Fig. $1, f\left(Q_{i}, P_{i}\right) \leqq Q_{i}$ for all $P_{i}$; i.e., bidder $i$ 's policy can never cause an increase in the trade's policy. If the initial trade policy, $Q_{i}$, is above the static equilibrium value, $P^{*}$, that satisfies (19), then there is no problem. However, if $Q_{i}$ is below $P^{*}$, then the equilibrium can never be 
reached. Any deviation by an optimizing bidder from the trade policy will only lead the members of the trade to lower their bids and will thus move them further from the "equilibrium." In this range, the model is analogous to the 'kinked demand curve' model of oligopoly theory, in which competitors match price decreases but not price increases.

The second reaction function we consider is

$$
f\left(Q_{i}, P_{i}\right)=Q_{i}+\alpha p_{i}\left(P_{i}-Q_{i}\right),
$$

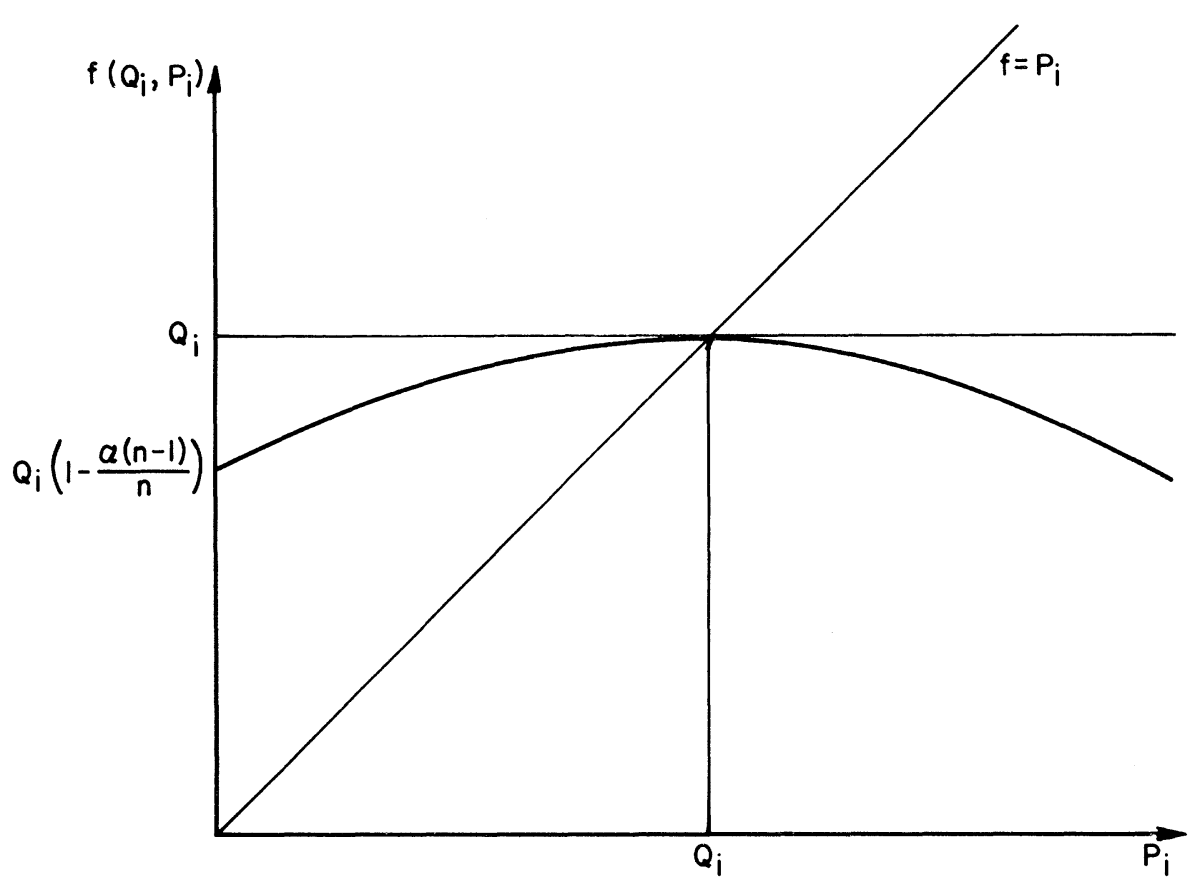

Fig. 1. $f\left(Q_{i}, P_{i}\right)=Q_{i}-\alpha\left[(1 / n)-p_{i}\right]\left(P_{i}-Q_{i}\right)$

where $Q_{i}, P_{i}, \alpha$, and $p_{i}$, are as in (16). This definition corresponds to an assumption that the trade reaction to a deviation from trade policy is proportional to the deviation and to the likelihood of the deviating bidder's winning the auction. It clearly satisfies (12). This reaction function considered as a function of $P_{i}$ for given $Q_{i}$ is illustrated in Fig. 2. If we again assume $P_{i}=0$ implies $p_{i}=1$, the function starts with $f\left(Q_{i}, 0\right)=$ $(1-\alpha) Q_{i}$. It then rises (falls for $\alpha<0$ ) with a slope that starts at $\alpha$ and decreases to $\alpha / n$ by the time $P_{i}$ has been increased to $Q_{i}$. At this point, $f\left(Q_{i}, Q_{i}\right)=Q_{i}$. It continues to increase (decrease if $\alpha<0$ ) until it reaches an extremum and then approaches $Q_{i}$ asymptotically for large $P_{i}$. To ensure that $f$ is positive for every positive $P_{i}$, we must restrict $\alpha$ to be less 
than unity. Furthermore, since in the identical bidders case negative values of $\alpha$ can be shown to result in an unstable equilibrium, we shall restrict $\alpha$ to be positive.

Again in the case of identical bidders, an equilibrium policy implies $Q_{i}=P_{i}=P^{*}$ and $p_{i}=1 / n$. Thus for the reaction function (20)

and

$$
\partial f(\xi, \eta) /\left.\partial \eta\right|_{\xi=\eta=P^{*}}=\alpha / n
$$

$$
\partial f(\xi, \eta) /\left.\partial \xi\right|_{\xi=\eta=P^{*}}=1-\alpha / n .
$$

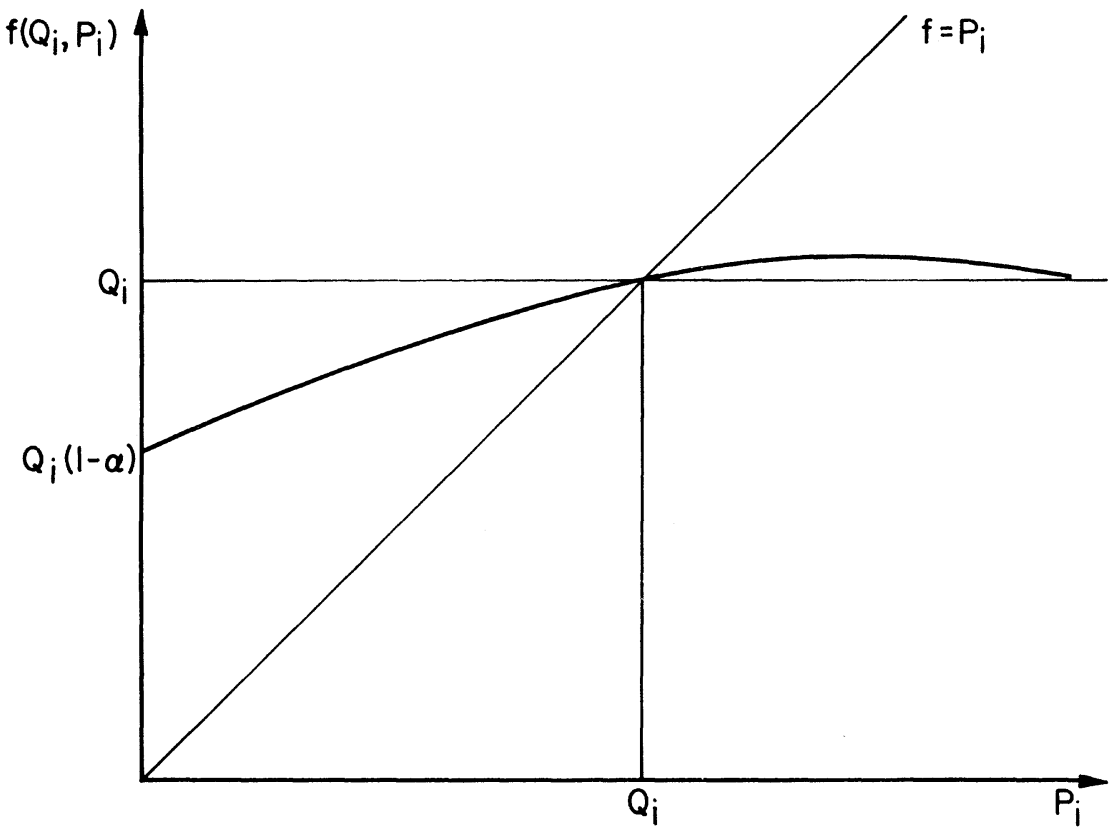

Fig. 2. $f\left(Q_{i}, P_{i}\right)=Q_{i}+\alpha p_{i}\left(P_{i}-Q_{i}\right)$

Substituting (21) and (22) in (13) yields the equilibrium condition

$$
\left.\{[\partial E(\xi, \eta) / \partial \eta][1-D(1-\alpha / n)]+[\partial E(\xi, \eta) / \partial \xi] D \alpha / n\}\right|_{\xi=\eta=P^{\bullet}}=0 .
$$

In this case the sequential auction equilibrium satisfying (23) is generally different from the one-shot equilibrium mentioned earlier. Two exceptional situations, in which (23) reduces to (19) and hence the two equilibriums are the same, occur when $D=0$ or $\alpha / n=0$. In the first case the bidders disregard future payoffs and thus behave as in a one-shot situation. In the second case the trade is insensitive to individual policies, either because $\alpha=0$ or because the number of bidders, $n$, is large. Thus, an individual bidder should not worry about his impact on the trade and can behave as in a one-shot auction. 
Substituting (21) and (22) in (15) yields the stability condition for the equilibrium policy satisfying (23). This condition is

$$
d \hat{P}(\xi) /\left.d \xi\right|_{\xi=P^{*}}<1 \text {. }
$$

Since $\hat{P}\left(P^{*}\right)=P^{*},(24)$ implies that $P^{*}$ is a stable policy if near $P^{*}$ the optimal policy of each bidder deviates from $P^{*}$ less than his estimate of the trade policy. A rigorous proof that this condition is satisfied involves the specific form of the reward function. However, in general, one can expect that if the trade bids very aggressively, bidder $i$ cannot make money in this auction. Thus his optimal strategy will be to lose the bid by bidding very unaggressively. On the other hand, if the trade bids unaggressively, he should do his best to win the bid by bidding more aggressively than the trade. If we assume $\hat{P}$ is a continuous function, the above implies that as $Q$ increases, $\hat{P}(Q)$ crosses the line $f=P_{i}$ from above. Thus its slope at $\hat{P}\left(P^{*}\right)=P^{*}$ is less than unity, as required by condition $(24)$.

\section{A PARTICULAR MODEL}

In this section we generalize to the case of sequential auctions some of the results obtained by Rothkopf ${ }^{[9]}$ for a one-shot model. Reference 9 describes a model of a competitive auction in which there are $n$ bidders, each with the same cost $c$ of doing a job. It is assumed that each bidder independently makes an unbiased estimate of his cost and then multiplies his estimate by a factor $P_{i}$ in order to arrive at his bid. The bidders' independent cost estimates are each assumed to come from a two-parameter Weibull distribution with spread parameter $m$. Under these circumstances, the expected profit from this auction for bidder $i$ is given by

where

$$
E_{i}\left(Q_{i}, P_{i}\right)=c p_{i}\left(P_{i} p_{i}^{-1 / m}-1\right),
$$

and

$$
p_{i}=1 /\left[1+(n-1)\left(P_{i} / Q_{i}\right)^{m}\right]
$$

$$
Q_{i}=\left[(n-1)^{-1} \sum_{j=1, j \neq i}^{j=n}\left(1 / P_{j}{ }^{m}\right)\right]^{-1 / m} .
$$

The quantity $p_{i}$ in (25) is the probability that bidder $i$ wins the auction, while the term in the parentheses times $c$ represents his expected profit if he wins.

Suppose now that the one-shot situation described above recurs at fixed time intervals and that each bidder has to consider the effect of his present bid on his future payoffs. In particular, we shall assume as before that bidder $i$ 's objective is to maximize his present value of future rewards with discount factor $D_{i}$. Clearly, the reward function (25) satisfies condition (1). We also assume the reaction function that is given by (20). Thus the identical bidders' equilibrium policy can be derived from (23) and (24). 
This results in

$$
P^{*}=m(n-1) n^{1 / m} /[m(n-1)-1-F],
$$

where $F$ is defined as $F=\alpha D /(1-D)$. For $F=0,(28)$ reduces to the oneshot equilibrium policy obtained in Rothkopf. ${ }^{[9]}$ This result is consistent with the observation made in Section 3 , that for $D=0$ or $\alpha=0$ (23) reduces to the one-shot equilibrium condition.

As $F$ is increased, $P^{*}$ increases until the equilibrium disintegrates at $F=m(n-1)-1$. Therefore we must restrict $\alpha$ to $\alpha<[m(n-1)-1]$ $\cdot(1-D) / D$. At equilibrium, the expected value of the winning bid will be

$$
c P^{*} n^{-1 / m}=c\{1+(1+F) /[m(n-1)-1-F]\},
$$

and the expected profit in each auction of each of the $n$ bidders will be

$$
E\left(P^{*}, P^{*}\right)=\left[c P^{*} n^{-1 / m}-c\right] / n=(c / n)(1+F) /[m(n-1)-1-F] .
$$

If the competition begins with equilibrium strategies, the present value of a bidder's profits in the present and all future auctions is given by $V\left(P^{*}\right)=$ $E\left(P^{*}, P^{*}\right) /(1-D)$. Obviously, the bidders are better off at equilibrium if the trade reacts strongly to price cutting (i.e., if $\alpha$ is large) than if it does not. The degree of reaction will probably depend on a number of institutional factors not fully represented by the model. These include the speed and certainty with which competitors can discern a policy change and the extent to which the competitors in one auction are likely to be the same as the competitors in the succeeding auctions.

\section{DISCUSSION AND CONCLUSIONS}

This paper accomplishes two things. First of all, we have derived an equation for an optimal infinite horizon bidding strategy. This equation is quite general. It can be used for a wide variety of assumptions about how competitive policy will change in reaction to the bidding policy of one bidder and how a bidder's profit in an auction depends upon his policy and that of his competitors.

Secondly, we have applied this model to a particular generalization of a symmetric, $n$-bidder, game-theoretic, one-shot bidding model. In doing so, we have changed the nature of the model. It is no longer strictly a game-theoretic model. We now assume each bidder to act on a behavioral assumption about how his competitors will react in the future to his present bidding policy. This assumption may not be satisfied by the actual behavior of the bidders even if each bidder uses a similar model. By an appropriate choice of our parameter $\alpha$, it may be possible to close the gap between what each bidder expects his competitors to do and what each competitor would do if he followed the advice of the model. This, however, has not yet been done. 
In spite of the possible gap between expected and actual reaction, we believe that the results given in equations (28), (29), and (30) provide insight into the effect of competitive reaction on optimal bidding policy and the profit to be paid by bid takers. Observe that the effect of the sequential nature of auctions depends only upon a factor that is the product of a parameter that measures the strength of competitive reaction and a simple function of the discount factor between auctions. Also note that the expected profit of the bidders is a nonlinear function of this factor. This dependence suggests a number of tactics that bid takers can pursue if they suspect that they are paying excessive profits to suppliers because of tacit collusion. They may be able to increase $m$ by reducing the uncertainty the bidders face; increase $n$ by bringing in additional bidders; decrease the discount factor $D$ by increasing the interval between successive auctions; and decrease $\alpha$ by changing institutional factors. These steps might include making it more difficult for the trade to react by keeping the amount of the winning bid secret and by frequently changing the list of invited bidders so that there is usually at least one new bidder present.

The model presented in Section 4 also has a tactical message for bidders. A bidder should restrain his aggressiveness in repetitive bidding situations if he thinks doing so will influence his competitors to behave less aggresssively in the future. Also, a bidder should try to convince his competitors that he will react to their policy changes.

\section{NOTE}

1. In addition, there are two works that deal with the problem of two bidders bidding sequentially for two items. ${ }^{[5,11]}$

\section{REFERENCES}

$\rightarrow$ Emil D. Attanasi, "Some Interpretations of Sequential Bid Pricing Strategies," Management Sci. 20, 1424-1427 (1974).

2. Bani Prasad Banerjeg and Prabir Kumar Ghosh, "A Problem of Sequential Competitive Biddings," in Advancing Frontiers in Operational Research, pp 215-225, H. S. Subba Rao, N. K. Jaiswal, and A. Ghosal (eds.) Hindustan Publishing Corp., Delhi, 1969.

3. G. M. Brofmser, "Competitive Bidding in the Construction Industry," Ph.D. Dissertation, Stanford University, 1968.

4. Arthur E. Bryson, Jr. and Yu-Chi Ho, Applied Optimal Control. Ginn and Co., Waltham, Mass., 1969.

5. K. Chen and K. H. Bhavnani, "An Investigation of Two-Person Competitive Bidding Strategies," IEEE Trans. on Systems, Man, and Cybernetics 4, 117-121 (1974).

6. Charles S. Knode and Lloyd A. Swanson, "A Dynamic Programming Approach to Competitive Bidding with Resource Constraints Including Costs for Over and Under Commitment," School of Management, Syracuse University, 1973; presented at 44th ORSA meeting. 
$\rightarrow$ K. D. Kortanek, J. V. Soden, and D. Sodaro, "Profit Analysis and Sequential Bid Pricing Models," Management Sci. 20, 396-417 (1973).

8. Randall Robinson, "Mathematical Models for Planning and Competitive Bidding by Semiconductor Manufacturers," Ph.D. Thesis, M.I.T., June, 1964.

$\rightarrow$ Michael H. Rothkopf, “A Model of Rational Competitive Bidding," Management Sci. 15, 362-373 (1969).

10. Michael H. Rothкорғ, "Contract Bid Development for the Small Businessman-A Dissent," Sloan Management Rev. 15, 65-67 (1973).

11. Maurice Sasieni, Arthur Yaspan, and Lawrence Friedman, Operations Research, Methods and Problems, Chapter 7, Wiley, New York, 1959.

$\rightarrow$ Robert M. Stark, "Competitive Bidding: A Comprehensive Bibliography," Opns. Res. 19, 484-490 (1971).

$\rightarrow$ Robert M. Stark and R. H. Mayer, Jr., "Some Multi-Contract Decision Theoretic Competitive Bidding Models," Opns. Res. 19, 469-483 (1971).

14. Thomas P. Tytula, "A Decision Theoretic Solution for a Bidding-Work Loading Game," Army Missile Command, Redstone Arsenal, Systems Engineering and Integration Office, Report RC-TR-1, February, 1972.

15. LeONARD H. ZACKs, "Queuing Theoretic Analysis of Contractors' Sequential Bidding Problems I and II," P-4412/1, 2, The Rand Corporation, Santa Monica, California, 1970. 